IdeAs

Idées d'Amériques

$10 \mid 2017$

États-Unis / Cuba : une nouvelle donne?

Tony Smith. Why Wilson Matters : The Origin of American Liberal Internationalism and Its Crisis Today. Princeton and Oxford: Princeton University Press, 2017. $332 \mathrm{p}$.

Princeton and Oxford : Princeton University Press, 2017. 332 p.

\title{
Serge Ricard
}

\section{OpenEdition \\ Journals}

\section{Édition électronique}

URL : https://journals.openedition.org/ideas/2185

DOI : $10.4000 /$ ideas. 2185

ISSN : 1950-5701

Éditeur

Institut des Amériques

\section{Référence électronique}

Serge Ricard, «Tony Smith. Why Wilson Matters : The Origin of American Liberal Internationalism and Its

Crisis Today. Princeton and Oxford : Princeton University Press, 2017. 332 p. », IdeAs [En ligne], 10 I

2017, mis en ligne le 18 décembre 2017, consulté le 19 octobre 2022. URL : http://

journals.openedition.org/ideas/2185; DOI : https://doi.org/10.4000/ideas.2185

Ce document a été généré automatiquement le 19 octobre 2022.

\section{(c) (†) $\ominus$}

Creative Commons - Attribution - Pas d'Utilisation Commerciale - Pas de Modification 4.0 International - CC BY-NC-ND 4.0

https://creativecommons.org/licenses/by-nc-nd/4.0/ 


\section{Tony Smith. Why Wilson Matters : The Origin of American Liberal Internationalism and Its Crisis Today. Princeton and Oxford : Princeton University Press, 2017. $332 \mathrm{p}$.}

Princeton and Oxford : Princeton University Press, 2017. 332 p.

\section{Serge Ricard}

\section{RÉFÉRENCE}

Tony Smith. Why Wilson Matters: The Origin of American Liberal Internationalism and Its Crisis Today. Princeton and Oxford : Princeton University Press, 2017. 332 p.

1 Le livre que Tony Smith consacre au "wilsonisme », connu par ailleurs sous le nom d'«internationalisme libéral», se déroule en deux temps: d'abord l'analyse des origines de la doctrine telle qu'elle se définit sous la plume du $28^{\mathrm{e}}$ président des ÉtatsUnis; ensuite sa résurgence sous Franklin Roosevelt et le rôle essentiel qu'elle a joué dans la conduite de la politique étrangère américaine de Pearl Harbor à l'effondrement de l'Union soviétique, puis le passage dans les années 1990, selon les termes de l'auteur, du stade « hégémonique » de l'ordre états-unien à sa phase « impérialiste » ou " néowilsonienne ». Ironie de l'histoire récente, le nouvel hôte de la Maison-Blanche, Donald Trump, prend le contre-pied de cette politique et tourne le dos à l'exceptionnalisme et au messianisme démocratique jusque-là invoqués pour justifier l'instauration d'une Pax Americana.

2 Smith entreprend éloquemment de raviver une tradition dont il s'est souvent montré dans ses travaux antérieurs fin exégète et ardent laudateur. Il s'appuie méticuleusement sur les écrits de Wilson pour montrer qu'au moment de son accession 
à la magistrature suprême ce dernier dispose d'un corps de doctrine cohérent, fruit de trois décennies de réflexion sur l'histoire américaine et britannique, le parlementarisme et la démocratie, à laquelle se mêle l'influence du presbytérianisme. Sa vision politique, pétrie d'« anglo-teutonnisme » et de darwinisme, se révèle en fait extrêmement modérée et réaliste et frappe par son idéalisme mesuré. L'établissement de la démocratie ne se fait pas ex nihilo; il nécessite un long apprentissage. La Révolution française avec sa tabula rasa et ses excès fait figure de contre-exemple et de repoussoir.

3 Selon Tony Smith, les concepts-clés du wilsonisme, pensés comme facteurs de paix et de stabilité dans le monde, sont au nombre de quatre: libre-échange et intégration économique, multilatéralisme assurant une "sécurité collective ", leadership éclairé de l'Amérique et, surtout, coopération entre les démocraties. Cependant, avant de théoriser sa vision des relations internationales, dont il voit l'expansion de la démocratie comme le but ultime, Wilson cède au tournant du siècle aux sirènes de l'expansionnisme civilisateur cher à Théodore Roosevelt, que Smith dénomme « impérialisme progressiste », à savoir cette " promotion de la démocratie » par la force mise en pratique avec des bonheurs divers aux Philippines au lendemain de la guerre hispano-américaine, puis, sous sa présidence, au Mexique, en Haïti et à SaintDomingue. L'élaboration du traité panaméricain de 1915-1916 conduit Wilson, plus kantien que hobbesien, à préciser sa conception du multilatéralisme comme fondement de la sécurité collective et initiateur de démocratie et de paix. Cette vision vient à maturité lorsqu'il fait face au conflit mondial. Elle a été ébauchée dès 1910, il faut le rappeler, par le premier Roosevelt dans son discours devant le comité Nobel de la Paix.

4 Avec la Société des Nations, Wilson entend alors bâtir un nouvel ordre mondial substituant au traditionnel équilibre des puissances la sécurité collective. Sa démarche consiste à transposer sur la scène mondiale sa réflexion sur la démocratie en Amérique, sa conviction qu'un gouvernement doit tenir son pouvoir du consentement des gouvernés, son anti-impérialisme et, comme le montrera son attitude envers l'Allemagne vaincue et la révolution bolchevique, sa certitude qu'un peuple culturellement et racialement homogène peut prendre son destin en main et évoluer vers une société de liberté - à condition qu'il dispose de citoyens engagés, dotés d'esprit civique, d'une élite dirigeante favorable à un État de droit et d'une classe moyenne soucieuse de voir ses aspirations prises en compte - mais qu'il est vain de vouloir stopper un mouvement révolutionnaire par l'envoi de troupes étrangères.

5 Smith s'attache ainsi à démontrer que la promotion de la démocratie et, partant, de la paix dans le monde est au cœur du projet wilsonien; pierre de touche de l'internationalisme libéral américain, elle est indispensable à l'établissement, sous l'égide de l'Amérique, d'un système certes bénéfique pour tous, mais dont le but premier est avant tout de garantir la sécurité des États-Unis et de protéger leurs intérêts globaux. Comme il apparaît dans la deuxième partie de l'ouvrage, consacrée au "wilsonisme sans Wilson ", le souci de l'auteur est de souligner l'extraordinaire succès et la pérennité d'une vision ambitieuse pour l'Amérique, mais également de corriger un certain nombre de malentendus et d'erreurs d'interprétation. La principale, au dire de Tony Smith, tient aux qualificatifs accolés au wilsonisme - idéaliste, moralisateur, utopique, porteur d'un impérialisme messianique - par nombre de spécialistes de la politique étrangère américaine. C'est faire peu de cas, selon, lui des écrits universitaires de Wilson, de son bilan présidentiel et des espoirs limités qui animaient sa vision 
prudente des progrès de la démocratie. La preuve de son adéquation au monde du XX siècle est que nonobstant son échec en 1920 et durant la décennie qui suit, l'internationalisme wilsonien retrouve son attrait lorsque éclate la Deuxième Guerre mondiale, d'autant plus que les hommes alors au pouvoir ont connu et servi le $28^{\mathrm{e}}$ président. L'héritage wilsonien prend alors de multiples formes, notamment l'instauration de 1941 à 1951 des premiers organismes internationaux et, surtout, l'occupation et la démocratisation de l'Allemagne et du Japon, deux réussites majeures dues en grande partie au passé et aux qualités de ces deux peuples - la leçon du wilsonisme, oubliée en Iraq en 2003, étant que la démocratie ne peut fleurir à partir de rien.

6 La guerre froide voit l'avènement de l'endiguement du communisme et à cette fin l'alliance avec des régimes antidémocratiques, au nom d'une doctrine "réaliste » et non point " libérale ", ce qui n'entache en rien aux yeux de Smith l'héritage wilsonien car les deux approches étaient complémentaires et Wilson lui-même était un « réaliste libéral ». Les internationalistes libéraux pendant cette période manifestaient d'ailleurs un optimisme prudent. Ces régimes autoritaires pouvaient éventuellement être influencés dans le sens d'un assouplissement. Les tensions entre réalistes et libéraux ne manqueront pas, marquées par des excès d'un côté (Iran en 1953, Guatemala en 1954, Chili en 1973) et des naïvetés de l'autre (Kennedy et l'Alliance pour le progrès, Carter et les droits de l'homme), les échecs tenant à ce que Wilson voyait comme essentiel dans la mutation démocratique, au-delà de l'engagement de Washington : le caractère et la nature d'un peuple. Cette donnée peut expliquer les victoires inattendues du « libéralisme » au Portugal, en Espagne et en Grèce entre 1975 et 1985, succès qui consoleront des déboires encourus en Amérique latine et en Asie du Sud-Est.

7 La présidence de Ronald Reagan avec sa politique anticommuniste agressive et sa réaction positive aux ouvertures de Mikhaïl Gorbatchev constitue une transition entre la fin de la guerre froide et les révisions apportées à la doctrine internationaliste libérale par les néo-conservateurs dans les années 1990. Démocratie, ouverture des marchés, multilatéralisme, leadership américain - l'esprit du wilsonisme se maintient, mais moins vigoureusement, avec les présidents George H. W. Bush et Bill Clinton. La chute de l'Union soviétique en 1991 et l'optimisme qu'elle engendre dans le camp libéral entraînent la formulation de nouveaux concepts par des néo-libéraux démocrates et leur diffusion par des néo-conservateurs républicains; ainsi naît le «néo-wilsonisme» qui ouvre à l'internationalisme libéral sa phase «impérialiste ». Smith entreprend dans le chapitre six une sévère analyse théorique, très fouillée, de l'éclosion et du développement dans le milieu universitaire et les cercles du pouvoir de théories néo-wilsoniennes qui vont dominer à partir de 2001, notamment avec la notion de "guerre juste», le droit d'ingérence et la guerre préventive de la « doctrine Bush ». Il note également une myopie aux graves conséquences dans les rapports officiels, le désintérêt pour le matériau humain et le contexte local des expériences de "promotion de la démocratie ", d'où les réactions de rejet des valeurs libérales dans le monde arabe et le choc de civilisations ainsi provoqué.

8 Il termine par un survol de la pratique néo-wilsonienne sous George W. Bush et Barack Obama prétextant « l'attrait universel de la démocratie », un « devoir de protection » et le fait que "nos intérêts et nos valeurs ne font qu'un"; il souligne leurs échecs l'invasion de l'Iraq par Bush et la tragique déstabilisation du Moyen-Orient au lieu du remodelage annoncé, la faiblesse de la politique d'Obama en Libye et en Syrie, ses 
mauvais choix lors du «printemps arabe » et sa diplomatie incantatoire (à l'exception de la normalisation des relations avec Cuba et de l'accord sur le nucléaire iranien qui n'étaient pas des initiatives strictement néo-wilsoniennes), le bourbier afghan dans les deux cas - et conclut au dévoiement du wilsonisme et à la nécessité d'un retour aux sources. La transformation de l'internationalisme libéral en une idéologie impérialiste dans les années 1990 a mis en péril les valeurs qu'il prétend défendre; il n'est que de voir les désastres causés en leur nom dans le monde musulman de l'Afrique du Nord à l'Afghanistan.

9 Au terme d'une recherche approfondie, mise en valeur par une argumentation serrée et un texte dense, mais au prix de quelques répétitions, Tony Smith nous livre une étude magistrale, du wilsonisme, de sa cohérence, de son impact sur les relations internationales, de l'usage qu'en firent les successeurs du $28^{\mathrm{e}}$ président, de ses dévoiements et des effets bénéfiques que pourrait produire un retour à la doctrine initiale dont les enseignements restent encore mal connus. Son livre est également une indispensable mise en perspective d'un siècle de politique étrangère américaine.

\section{AUTEURS}

\section{SERGE RICARD}

Professeur émérite d'histoire et civilisation américaines à la Sorbonne Nouvelle (Université

Paris III) 